\title{
The Impact of Reabsorption on the Emission Spectra and Recombination Dynamics of Hybrid Perovskite Single Crystals
}

Hiba Diab, ${ }_{1}^{1}$ Christophe Arnold, ${ }^{2}$ Ferdinand Lédée, ${ }^{1}$ Gaëlle Trippé-Allard, ${ }^{1}$ Géraud Delport, ${ }^{1}$ Christèle Vilar, ${ }^{2}$ Fabien Bretenaker, ${ }^{1}$ Julien Barjon, ${ }^{2}$ Jean-Sébastien Lauret, ${ }^{1}$ Emmanuelle Deleporte $^{1}$ and Damien Garrot ${ }^{2}$

Laboratoire Aimé Cotton, CNRS, Univ. Paris-Sud, ENS Paris-Saclay, Université Paris-Saclay, 91405 Orsay Groupe d'Etude de la Matière Condensée, CNRS, Université de Versailles Saint Quentin-En-Yvelines,

Université Paris-Saclay, 45Avenue des Etats-Unis, 78035,Versailles.damien.garrot@uvsq.fr

Understanding the surface properties of hybrid perovskite is crucial to improve the efficiency of devices. Different studies suggest the existence of important differences between the surface and bulk photophysical properties of hybrid perovskites. ${ }^{1,2}$ Recently, an increase of the optical band gap of the surface layer relative to the bulk has been suggested for $\mathrm{CH}_{3} \mathrm{NH}_{3} \mathrm{PbBr}_{3}$ single crystals, due to ion migration. ${ }^{2}$ Such effect would have a detrimental impact on device performance.

In this study, we have investigated the surface and bulk properties of $\mathrm{CH}_{3} \mathrm{NH}_{3} \mathrm{PbBr}_{3}$ single crystals with a combination of cathodoluminescence (CL), steady-state and time-resolved photoluminescence (PL) spectroscopy. Firstly, depth-resolved CL has been used to probe the near surface region on depth ranging from a few nanometers to several micrometers. Secondly, we have studied the transmitted PL through different thicknesses. In both cases, experimental emission spectra were compared with simulated spectra, taking into account reabsorption effect. The results reveal the strong impact of reabsorption on the emission of hybrid perovskites. Reabsorption effect explains mainly the large variation of the emission spectra reported for hybrid perovskite single crystals, as well as the apparent differences between bulk and surface properties. In addition, we show that hybrid perovskite single crystals, even with millimeter size, are partially transparent to their own luminescence. The transmitted PL presents a long rising time and a lengthening of its decay due to photon recycling and light-trapping. ${ }^{3}$

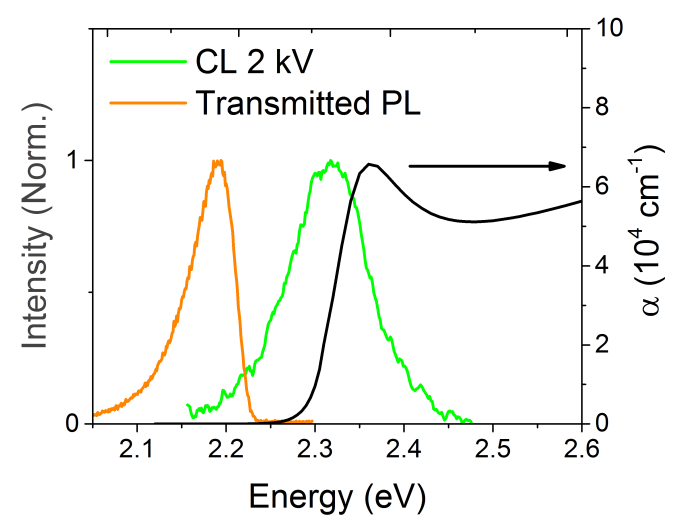

Figure 1: CL spectrum at $2 \mathrm{kV}$ (green), transmitted PL through a $600 \mu \mathrm{m}$ thickness (orange) and absorption coefficient (black) of $\mathrm{CH}_{3} \mathrm{NH}_{3} \mathrm{PbBr}_{3}$ single crystals. ${ }^{4}$

\footnotetext{
$\overline{1 .} \quad$ Wu, B. et al. Discerning the Surface and Bulk Recombination Kinetics of Organic-Inorganic Halide Perovskite Single Crystals. Adv. Energy Mater. 6, 1600551 (2016).

2. Sarmah, S. P. et al. Double Charged Surface Layers in Lead Halide Perovskite Crystals. Nano Lett. acs.nanolett.7b00031 (2017). doi:10.1021/acs.nanolett.7b00031

3. Diab, H. et al. The impact of Reabsorption on the Emission Spectra and Recombination Dynamics of Hybrid Perovskite Single Crystals. Submitted (2017).

4. Park, J.-S. et al. Electronic Structure and Optical Properties of $\alpha-C H 3 N H 3 P b B r 3$ Perovskite Single Crystal. J. Phys. Chem. Lett. 6, 4304-4308 (2015).
}

The project leading to this application has received funding from the European Union's Horizon 2020 research and innovation programme under grant agreement No 687008 (GOTSolar). 